\title{
Matrix-Ball Construction of affine Robinson-Schensted correspondence
}

\author{
Michael Chmutov|", Pavlo Pylyavskyy|", and Elena Yudovina
}

Department of Mathematics, University of Minnesota, Minneapolis, MN 55455, USA

\begin{abstract}
In his study of Kazhdan-Lusztig cells in affine type $A$, Shi has introduced an affine analog of RobinsonSchensted correspondence. We generalize the Matrix-Ball Construction of Viennot and Fulton to give a more combinatorial realization of Shi's algorithm. As a biproduct, we also give a way to realize the affine correspondence via the usual Robinson-Schensted bumping algorithm. Next, inspired by Honeywill, we extend the algorithm to a bijection between extended affine symmetric group and triples $(P, Q, \rho)$ where $P$ and $Q$ are tabloids and $\rho$ is a dominant weight. The weights $\rho$ get a natural interpretation in terms of the Affine Matrix-Ball Construction. Finally, we prove that fibers of the inverse map possess a Weyl group symmetry, explaining the dominance condition on weights.

Résumé. Dans son étude des cellules de Kazhdan-Lusztig de type $A$ affine, Shi a introduit un analogue affine de la correspondance de Robinson-Schensted. On généralise la Construction Matrice-Ballon de Viennot et Fulton afin de donner une réalisation plus combinatoire de l'algorithme de Shi. Comme sous-produit, on montre aussi un moyen de réaliser la correspondance affine par l'algorithme familier de l'insertion de Schensted. Puis, inspiré par Honeywill, on étend l'algorithme vers une bijection entre les éléments de la groupe affine symétrique étendue et les triples $(P, Q, \rho)$ où $P$ et $Q$ sont des tabloids et $\rho$ est un poids. Les poids $\rho$ obtiennent une interprétation naturelle en vue de la Construction Affine Matrice-Ballon. Finalement, on établit que les fibres de l'application inverse possèdent une symétrie du groupe de Weyl, ce qui explique la dominance des poids.
\end{abstract}

Keywords. Robinson-Schensted correspondence, Affine symmetric group, Kazhdan-Lusztig theory

\section{Introduction}

This is an extended abstract; the full version of the paper is [CPY15].

\subsection{Cells in Kazhdan-Lusztig theory}

In their groundbreaking paper [KL89] Kazhdan and Lusztig gave an approach to the representation theory of Hecke algebras. Since then this approach, now called called Kazhdan-Lusztig theory, has been significantly developed. Of particular importance in it are the notions of left cells, right cells, and two-sided cells. Left (resp. right) cells are equivalence classes corresponding to a certain pre-order $\leq_{L}\left(\right.$ resp. $\left.\leq_{R}\right)$ on a Coxeter group $W$. Two-sided cells are larger classes with both left and right equivalences allowed.

${ }^{\dagger}$ partially supported by NSF grants DMS-1148634 and DMS-1503119

${ }^{\ddagger}$ partially supported by NSF grants DMS-1148634, DMS-1351590, and Sloan Fellowship

1365-8050 @ 2016 Discrete Mathematics and Theoretical Computer Science (DMTCS), Nancy, France 
In type $A$, i.e. when $W$ is a symmetric group, the Kazhdan-Lusztig cell structure corresponds to the Robinson-Schensted correspondence, a bijective correspondence between elements of the symmetric group and pairs of standard Young tableaux of the same shape. It is well known ([BV82], [KL89], [GM88], [Ari99]) that two permutations lie in the same left (resp. right) cell whenever they have the same recording tableau $\bar{Q}$ (resp. insertion tableau $\bar{P}$ ), and in the same two-sided cell whenever the four tableaux have the same shape.

\subsection{Affine type $A$}

For $W$ of affine type $A$, Shi has shown that the left (and right) Kazhdan-Lusztig cells correspond to tabloids ([Shi86]). These are equivalence classes of fillings of Young diagrams with the first several integers up to permuting elements within rows. The shape of these tabloids determines the two-sided cell. Furthermore, in [Shi91] he gave an algorithm for constructing the tabloid $P(w)$ corresponding to the left cell of $w \in W$. We refer to this algorithm as Shi's algorithm. The algorithm consists of several distinct sub-algorithms applied in a specific order, making it challenging to develop a direct intuitive connection between the permutation and the resulting tabloid.

\subsection{Goals}

Goal A. Find a description of the Shi correspondence which would generalize a known construction of the usual Robinson-Schensted correspondence. The Matrix-Ball Construction (MBC) turns out to work well for us. The construction is present in [Vie77], however we use the terminology from [Ful97]. We introduce an algorithm called Affine Matrix-Ball Construction (AMBC) which from an affine permutation $w$ produces two tabloids $(P(w), Q(w))$ of common shape $\lambda$, and $\rho \in \mathbb{Z}^{\ell(\lambda)}$ (see below for its purpose).

Theorem 1.1 The outcome of Shi's algorithm coincides with the tabloid $P(w)$ of AMBC.

Surprisingly, as a biproduct of our analysis we get a realization of the Shi correspondence in terms of the usual Robinson-Schensted bumping algorithm. AMBC has been implemented in Java ([Chm15]); we suggest the program to the reader for additional examples.

Goal B. Describe an extension of Shi's correspondence to a bijection in terms of AMBC. Since there are only finitely many pairs of tabloids of a given shape and $W$ is infinite, the map $w \mapsto(P, Q)$ cannot be an injection. One can, however, get a bijection if one adds a third piece to the data. Namely, there exists a bijection of the form $w \mapsto(P, Q, \rho)$, where $\rho$ is a dominant weight for a certain finite Weyl group. This was known since Lusztig's conjecture in [Lus89]. An explicit description of this correspondence was given by Honeywill [Hon05], relying on results of Xi [Xi02].

We describe the inverse map $(P, Q, \rho) \mapsto w$ for AMBC thus proving that indeed our map is a bijection. We leave the question of the exact relation between our weights and those of Honeywill for future research.

Throughout the paper we work with the extended affine symmetric group, as does Honeywill in [Hon05]. However we prove

Theorem 1.2 Suppose $w$ is an extended affine permutation and $(P, Q, \rho)$ is the output of AMBC. Then $w$ lies in the non-extended affine permutation group if and only if $\sum_{i} \rho_{i}=0$.

So one recovers all of the theory for the non-extended case.

Goal $C$. The inverse map $(P, Q, \rho) \mapsto w$ is defined for any (non necessarily dominant) weight; describe the Weyl group action on its fibers. We are unaware of anything similar to this theorem in the existing literature. 


\subsection{Notational preliminaries}

Let $n$ be a positive integer. Let $[n]:=\{1, \ldots, n\}$. For each $i \in Z$ denote by $\bar{i}$ the residue class $i+n \mathbb{Z}$. Let $\overline{[n]}:=\{\overline{1}, \ldots, \bar{n}\}$. Let $\widetilde{W}$ be the extended affine symmetric group of type $\widetilde{A}_{n-1}$; it consists of elements $w: \mathbb{Z} \rightarrow \mathbb{Z}$ such that $w(i+n)=w(i)+n$. The elements of $\widetilde{W}$ are called extended affine permutations, or just permutations. Let $\bar{W}$ be the affine symmetric group of type $\widetilde{A}_{n-1}$; it consists of elements $w \in \widetilde{W}$ such that $\sum_{i=1}^{n} w(i)=\frac{n(n+1)}{2}$.

A partial permutation is a pair $(U, w)$ where $U \subseteq \mathbb{Z}$ has the property that $(x \in U) \Leftrightarrow(x+n \in U)$ and $w: U \rightarrow \mathbb{Z}$ such that $w(i+n)=w(i)+n$. We will suppress the explicit mention of the subset $U$ in the notation and just refer to the partial permutation $w$.

We usually think of permutations in terms of pictures such as the one in Figure 1 More precisely, on the plane we draw an infinite matrix; the rows (resp. columns) are labeled by $\mathbb{Z}$, increasing downward (resp. to the right). If $w(i)=j$ then we place a ball in the $i$-th row and $j$-th column. For a partial permutation $w$, we denote by $\mathcal{B}_{w} \subset \mathbb{Z} \times \mathbb{Z}$ the collection of balls of $w$. To distinguish the 0 -th row, we put a solid red line between the 0 -th and 1 -st rows, and similarly for columns. We also put dashed red lines every $n$ rows and columns.

For an integer $k$ and a ball $b=(i, j)$ we will refer to the ball $b^{\prime}=(i+k n, j+k n)$ as the $k(n, n)$ translate of $b$. Two balls $b$ and $b^{\prime}$ are $(n, n)$-translates if for some $k$ one is a $k(n, n)$-translate of the other.

We will assign numbers to balls of permutations as well as to other cells of the matrix. For a partial permutation $w$, a numbering of $w$ is a function $d: \mathcal{B}_{w} \rightarrow \mathbb{Z}$. A numbering $d$ is semi-periodic with period $m$ if for any $b \in \mathcal{B}_{w}$ we have $d(b+(n, n))=d(b)+m$. When referring to a numbering in pictures, we will write $d(b)$ inside the ball $b$ as done in Figure 1 , where we show a semi-periodic numbering of period 3 .

We use compass directions (e.g. north, east, northeast, etc.) inside the matrix with north being toward the top of the page and east being toward the right of the page.

Sequences of cells going southeast and northwest will be particularly important to us. A path is a sequence $\left(b_{0}, \ldots, b_{k}\right)$ of cells such that for each $i, b_{i+1}$ is northwest of $b_{i}$. The number $k$ is referred to as the length or number of steps of the path.

\section{Affine Matrix Ball Construction}

\subsection{Proper numberings}

In MBC one began by numbering the balls of the permutation according to a certain rule. In this section we introduce a collection of numberings, called proper numberings, any of which can be used to produce the tabloids. In Section 2.3 we will choose a particular proper numbering to be used in AMBC.

Definition 2.1 A function $d: \mathcal{B}_{w} \rightarrow \mathbb{Z}$ is a proper numbering if it satisfies

- (Monotonicity) for any $b, b^{\prime} \in \mathcal{B}_{w}$, if b lies northwest of $b^{\prime}$ then $d(b)<d\left(b^{\prime}\right)$,

- (Continuity) for any $b^{\prime} \in \mathcal{B}_{w}$ there exists b northwest of it with $d(b)=d\left(b^{\prime}\right)-1$.

An example of a proper numbering is given in Figure 1 . The permutation is $w=[4,1,6,11,2,3]$ in the sense that $w(1)=4, w(2)=1$, etc. It is not apriori obvious that proper numberings exist; we will describe a construction of such a numbering in Section 2.3 

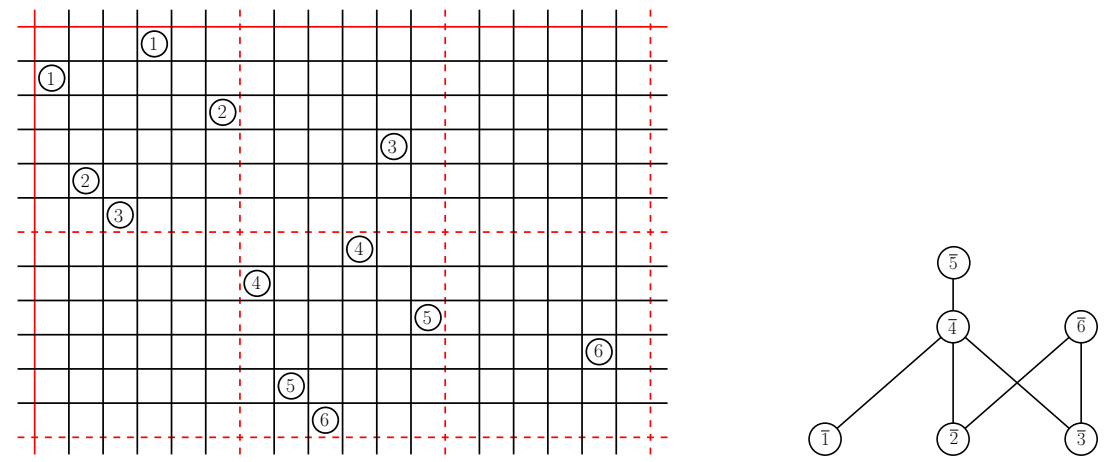

Fig. 1: On the left is a proper numbering for the (extended) affine permutation $[4,1,6,11,2,3]$. On the right is the Hasse diagram for the Shi poset of the same permutation.

\subsection{Shi poset}

Given a permutation $w \in \widetilde{W}$, Shi defined a (labeled) poset on $[n]$.

Definition 2.2 The (labeled) Shi poset $P_{w}$ associated with $w \in \widetilde{W}$ is the poset on $[n]$ with $i \leqslant_{S} j$ if either

$$
i>j \text { and } w(i)<w(j),
$$

or

$$
w(j)>w(i)+n .
$$

The element $i$ is labeled by the residue class $\overline{w(i)}$.

Example 2.3 For $w=[4,1,6,11,2,3]$, the Shi poset is shown in Figure 1$]$

We can now state the first major result about the structure theory of proper numberings.

Proposition 2.4 Any proper numbering is semi-periodic with period m equal to the width (maximal size of an antichain) of the Shi poset.

The numbering in Figure 1 is semi-periodic with period 3.

\subsection{Channels and channel numberings}

In this section we introduce a collection of proper numberings which both provides the first examples of them and plays the most important role in AMBC.

The projection map $\varphi_{w}: \mathcal{B}_{w} \rightarrow P_{w}$ is the map sending $(i, w(i))$ to the representative of $\bar{i}$ in $[n]$.

Definition 2.5 A channel is a preimage under the projection map of a longest antichain of the Shi poset.

The projections of two balls are incomparable in the Shi poset precisely when no two $(n, n)$-translates of these balls are southwest of each other. Thus any channel can be described as follows. Consider a ball $b$ and a path $B=\left(b_{0}=b, b_{1}, b_{2}, \ldots, b_{k}=b-(n, n)\right)$. If $k$ is as large as possible (i.e. $\left.k=m\right)$, then we can form a channel by taking all translates of $B$. Moreover, any channel can be obtained in this way. 

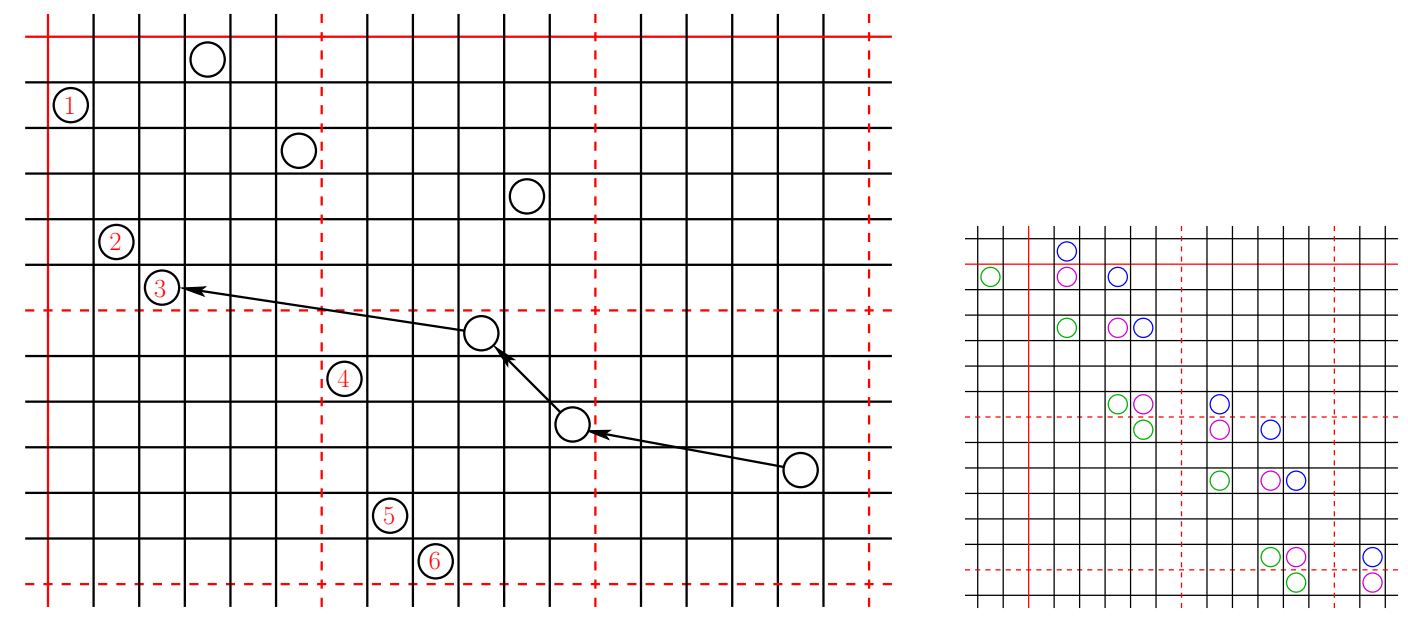

Fig. 2: On the left is a channel of $[4,1,6,11,2,3]$ with a proper numbering. The path shown has 3 steps and leads to a ball numbered 3 . Hence the worth of the path is 6 . On the right are some streams for $A=\{\overline{1}, \overline{3}, \overline{6}\}$ and $B=\{\overline{2}, \overline{4}, \overline{5}\}$.

Let $\mathcal{C}_{w}$ be the set of all channels for a given $w$. Fix $C \in \mathcal{C}_{w}$ and choose a proper numbering $\tilde{d}: C \rightarrow \mathbb{Z}$ of $C$ (there is an overall shift freedom). An example of such a numbering is shown in red in Figure 2 . Given a path $\left(b_{0}, b_{1}, \ldots, b_{k}\right)$ from a ball $b_{0}$ to $C$ (i.e. $b_{k} \in C$ ), we will refer to the number $\tilde{d}\left(b_{k}\right)+k$ as the worth of the path.

Definition 2.6 Suppose $C$ is a channel and $\tilde{d}$ is a proper numbering of it. Define the channel numbering of $\mathcal{B}_{w}$ by

$$
d_{w}^{C}(b):=\sup _{\left(b_{0}, b_{1}, \ldots, b_{k}\right)} \tilde{d}\left(b_{k}\right)+k,
$$

where the supremum is taken over all paths from $b$ to $C$.

It is clear that the numbering $d_{w}^{C}$ is proper if the supremum is always finite. This is indeed the case:

Proposition 2.7 Suppose $C$ is a channel of a partial permutation $w$ and $\tilde{d}: C \rightarrow \mathbb{Z}$ is a proper numbering. Then for any ball $b$, the set

$$
\left\{\tilde{d}\left(b_{k}\right)+k:\left(b_{0}, b_{1}, \ldots, b_{k}\right) \text { is a path from } b \text { to } C\right\}
$$

\section{is bounded above.}

Now we describe how to choose a distinguished channel for any $w$. Consider the following partial order on $\mathcal{C}_{w}$ (we will refer to it as the southwest partial ordering). A channel $C_{1}$ is southwest of a channel $C_{2}$ if for every element of $C_{1}$ there exists an element of $C_{2}$ weakly northeast of it.

Proposition 2.8 The above partial ordering has a least element and a greatest element.

Thus there exists a southwest channel and a northeast channel. In AMBC we will be using the southwest channel numbering of balls. 


\subsection{Streams}

Let $A=\left\{\overline{a_{1}}, \ldots, \overline{a_{k}}\right\}$ and $B=\left\{\overline{b_{1}}, \ldots, \overline{b_{k}}\right\}$ be two subsets of $\overline{[n]}$ of the same size. Let $\mathfrak{s t}(A, B)$ be a collection of subsets of $\mathbb{Z} \times \mathbb{Z}$ such that for any $S \in \mathfrak{s t}(A, B)$ all of the following hold

- if $(i, j) \in S$ then $(i, j) \pm(n, n) \in S$;

- for any two elements of $S$ one is southeast of the other;

- $A=\{\bar{i}:(i, j) \in S\}$ and $B=\{\bar{j}:(i, j) \in S\}$.

We refer to elements of $\mathfrak{s t}(A, B)$ obtained in this way as streams. There are infinitely many $(A, B)$ streams, we classify them in the following way.

Lemma 2.9 There is a unique stream $\mathfrak{s t}_{0}(A, B) \in \mathfrak{s t}(A, B)$ such that there are exactly $k=|A|=|B|$ of $(i, j) \in \mathfrak{s t}_{0}(A, B)$ such that $1 \leq i, j \leq n$.

Now, let $\ldots,\left(i_{-1}, j_{-1}\right),\left(i_{0}, j_{0}\right),\left(i_{1}, j_{1}\right), \ldots$ be the list of elements of $\mathfrak{s t}_{0}(A, B)$ from northwest to southeast.

Proposition 2.10 For each $r \in \mathbb{Z}$ let $\mathfrak{s t}_{r}(A, B)=\left\{\left(i_{t}, j_{t+r}\right) \mid t \in \mathbb{Z}\right\}$. Then each $\mathfrak{s t}_{r}(A, B)$ is an $(A, B)$-stream, and all $(A, B)$-streams arise this way.

Example 2.11 Let $n=6, A=(1,3,6)$ and $B=(2,4,5)$. Then the streams $\mathfrak{s t}_{-1}(A, B), \mathfrak{s t}_{0}(A, B)$ and $\mathfrak{s t}_{1}(A, B)$ are shown in Figure 2

\subsection{The map}

Let $\lambda=\left(\lambda_{1}, \ldots, \lambda_{\ell}\right)$ be a partition of $n$. Let $\Omega$ be the set of all triples $(P, Q, \rho)$, where $P$ and $Q$ are tabloids of the same shape $\lambda$ of size $n$, and $\rho \in(\mathbb{Z})^{\ell}$. Note that the $i$-th row in an element of $\Omega$ describes a stream, $\mathfrak{s t}_{\rho_{i}}\left(P_{i}, Q_{i}\right)$.

Definition 2.12 Suppose $w$ is a partial permutation and $d: \mathcal{B}_{w} \rightarrow \mathbb{Z}$ is a proper numbering. For each $i \in \mathbb{Z}$ form a Young diagram whose inner corners are the balls numbered $i$ (in Figure 3 these are drawn in magenta and green). Define $\mathrm{fw}_{d}(w)$ to be the permutation whose balls are the outer corners of the Young diagram. If $C$ is a channel, we write $\mathrm{fw}_{C}(w) f_{0} \mathrm{fw}_{d_{w}^{C}}(w)$. If $C$ was the southwest channel then we write $\mathrm{fw}(w)$ for $\mathrm{fw}_{C}(w)$.

Definition 2.13 Suppose $w$ is a partial permutation and $d: \mathcal{B}_{w} \rightarrow \mathbb{Z}$ is a proper numbering. Let $b_{r}=$ $\left(i_{r}, j_{r}\right)$, where $i_{r}$ is the row of the northern ball labeled $r$ and $j_{r}$ is the column of the western ball labeled $r$ (in Figure 3 they are labeled by magenta $*$ 's). Then $\left\{b_{r}\right\}_{r \in \mathbb{Z}}$ forms a stream. This stream will be denoted $\mathfrak{s t}_{d}(w)$. If $C$ is a channel we will write $\mathfrak{s t}_{C}(w)$ for $\mathfrak{s t}_{d_{w}^{C}}(w)$. We will write $\mathfrak{s t}(w)$ when using the southwest channel numbering.

We are ready to define the map $\Phi: \widetilde{W} \rightarrow \Omega$. We refer to the algorithm below as Affine Matrix-Ball Construction (AMBC); it take $w \in \widetilde{W}$ as input and outputs a triple $(P, Q, \rho) \in \Omega$.

- Initialize $(P, Q, \rho)$ to $(\varnothing, \varnothing, \varnothing)$.

- Repeat until $w$ is the empty partial permutation: 


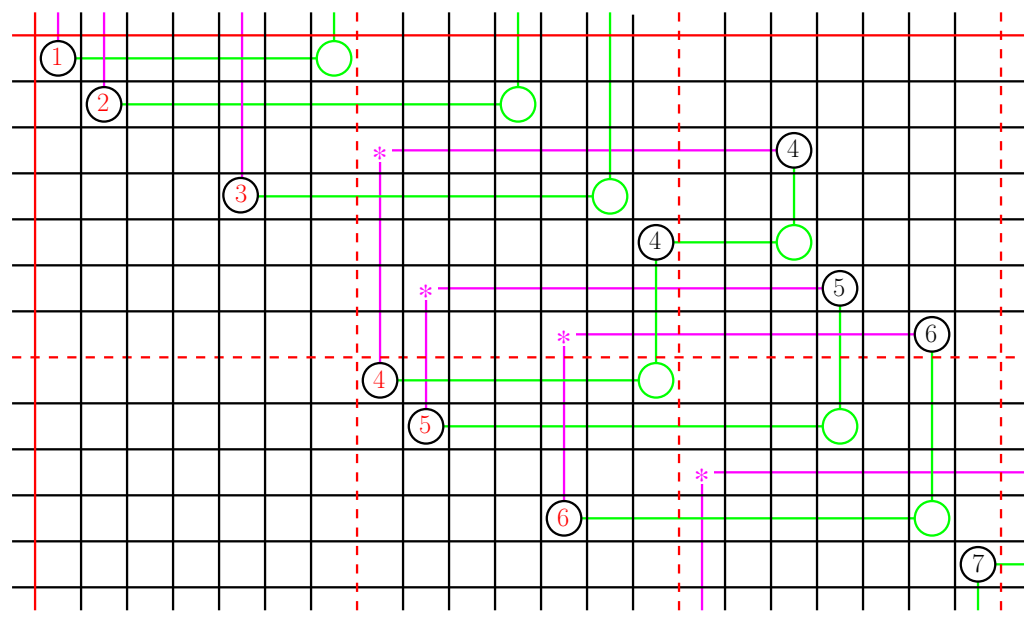

Fig. 3: First step of AMBC for $[1,2,17,5,14,18,20]$. The numbering shown is the SW channel numbering; the balls in the channel are numbered in red. The balls of $\mathrm{fw}(w)$ are shown in green. The cells $b_{r}$ denoted by *'s. At the end of this step we set the first row of $P$ to $\{\overline{1}, \overline{2}, \overline{5}\}$, the first row of $Q$ to $\{\overline{0}, \overline{3}, \overline{6}\}$, and the first row of $\rho$ to 3 .

- Record the data of $\mathfrak{s t}(w)$ in the next row of $P, Q$, and $\rho$.

- Reset $w$ to fw $(w)$.

Example 2.14 Consider $w=[1,2,17,5,14,18,20]$ for $n=7$. The first step of AMBC is shown in Figure 3. Proceeding in the same fashion results in

$$
(P, Q, \rho)=\left(\begin{array}{|l|l|l|}
\hline \overline{1} & \overline{2} & \overline{5} \\
\hline \overline{0} & \overline{4} & \overline{6} \\
\hline \overline{3} &
\end{array}, \quad \begin{array}{|l|l|l|}
\hline \overline{0} & \overline{3} & \overline{6} \\
\hline \overline{2} & \overline{4} & \overline{5} \\
\hline \overline{1} & & \\
\hline
\end{array}, \quad \begin{array}{|l|l|}
3 \\
\hline 3 \\
\hline 1
\end{array}\right)
$$

Proposition 2.15 The two tabloids $(P, Q)$ in the outcome do not change if we use an arbitrary proper numbering of balls at each step.

\section{Backward algorithm}

In this section we will introduce an algorithm that gives the inverse map $\Psi: \Omega \rightarrow \widetilde{W}$.

\subsection{Backward numberings}

First we explain a way to construct a numbering of the balls of a partial permutation with respect to a stream.

Definition 3.1 We say that a stream $S \in \mathfrak{s t}(A, B)$ is compatible with a partial permutation $w$ if $\mathcal{B}_{w} \cup S$ has at most one cell in each row and at most one cell in each column, and if $|A|=|B|$ is at least the width of $P_{w}$. 
Definition 3.2 Consider a partial permutation $w$ and a compatible stream $S$; choose a proper numbering of the stream. For each $b \in \mathcal{B}_{w}$ let $d_{0}(b)$ be the largest number of a stream element northwest of $b$. Then for any ball $b$ of $w$, define the backward numbering of $b$ by

$$
d(b)=\min _{\left(b_{0}, \ldots, b_{k}=b\right)} d_{0}\left(b_{0}\right)-k,
$$

where the minimum is taken over all paths ending at $b$.

\subsection{The map}

Now we are ready to describe the backward algorithm (map $\Psi$ ), which takes as input $(P, Q, \rho) \in \Omega$ and outputs $w \in \widetilde{W}$ :

- Initialize $w$ to the empty partial permutation.

- Repeat until $(P, Q, \rho)=(\varnothing, \varnothing, \varnothing)$ :

- Remove the last row of $P$ and call it $A$; remove the last row of $Q$ and call it $B$; remove the last row of $\rho$ and call it $r$. Give the stream $S:=\mathfrak{s t}_{r}(A, B)$ some proper numbering. Let $d: \mathcal{B}_{w} \rightarrow \mathbb{Z}$ be the backward numbering corresponding to $S$.

- For each $i \in \mathbb{Z}$ form a Young diagram with top-left corner at the cell of $S$ labeled $i$ and outer corners at the balls of $\mathcal{B}_{w}$ labeled $i$. Note that sometimes there are no balls labeled $i$; in this case we end up with the empty Young diagram which, by convention, has no outer corners, but has one inner corner.

- Consider the partial permutation $\mathrm{bk}_{S}(w)$ consisting of the inner corners of the Young diagram.

- Let $w=\operatorname{bk}_{S}(w)$

\section{Bijectivity}

In this section we describe the image of $\Phi$ as a subset of $\Omega$. This description will have the form: a collection of triples $(P, Q, \rho) \in \Omega$, where $\rho$ satisfies certain inequalities which depend on $P$ and $Q$. The primary goal is to describe the inequalities explicitly.

\subsection{Concurrent streams}

Definition 4.1 Let $S, T$ be two streams of the same flow and choose a proper numbering of $S$. Let $t$ be the partial permutation whose balls are the cells of $T$; assume $S$ is compatible with $t$. Let $d$ be the backward numbering of $t$ with respect to $S$. Then $T$ is concurrent to $S$ if there exists $i, j$ such that

- the ball $b$ numbered $i$ by $d$ is north of the cell of $S$ labeled $i+1$, and

- the ball $b^{\prime}$ numbered $j$ by $d$ is west of the cell of $S$ labeled $j+1$.

Proposition 4.2 Consider four sets $A, A^{\prime}, B, B^{\prime} \subset[n]$, all of the same size, with $A$ disjoint from $A^{\prime}$ and $B$ disjoint from $B^{\prime}$. Then there exists a unique $r \in \mathbb{Z}$ such that $\mathfrak{s t}_{r}\left(A^{\prime}, B^{\prime}\right)$ is concurrent to $\mathfrak{s t}_{0}(A, B)$. Moreover, for any $l, \mathfrak{s t}_{r+l}\left(A^{\prime}, B^{\prime}\right)$ is concurrent to $\mathfrak{s t}_{l}(A, B)$. 


\subsection{Dominant weights}

Suppose $(P, Q, \rho) \in \Omega$ and $P$ and $Q$ have shape $\lambda$. Let $r_{1}=0$. For each $2 \leqslant i \leqslant \ell(\lambda)$ : if $\lambda_{i}<\lambda_{i-1}$ then let $r_{i}=0$, otherwise let $r_{i}$ be the unique integer such that $\mathfrak{s t}_{r_{i}}\left(P_{i}, Q_{i}\right)$ is concurrent to $\mathfrak{s t}_{0}\left(P_{i-1}, Q_{i-1}\right)$.

Definition 4.3 Consider $(P, Q, \rho) \in \Omega$. Suppose $P$ and $Q$ have shape $\lambda$. Then $\rho$ is dominant if for each $1 \leqslant i<l(P)$ either $\lambda_{i}>\lambda_{i+1}$, or $\lambda_{i}=\lambda_{i+1}$ and $\rho_{i+1}-r_{i+1} \geqslant \rho_{i}-r_{i}$.

Let $\Omega_{d o m}:=\{(P, Q, \rho) \in \Omega: \rho$ is dominant $\}$. Then $\Phi$ is a bijection between $\widetilde{W}$ and $\Omega_{d o m}$.

Theorem 4.4 We have $\Phi(\widetilde{W}) \subseteq \Omega_{\text {dom. }}$. For any $w \in \widetilde{W}, \Psi(\Phi(w))=w$. Moreover, if $(P, Q, \rho) \in \Omega_{\text {dom }}$, then $\Phi(\Psi(P, Q, \rho))=(P, Q, \rho)$.

Remark 4.5 In the standard presentation of the Lie algebra $\mathfrak{s l}_{n}$, the dominant integral weights are increasing sequences of integers. Thus our dominant weights are actually located in some shift of the dominant chamber of a product of special linear Lie algebras.

\section{Weyl group action}

In this section we describe the result of $\Phi \circ \Psi$ on a non-dominant weight (and the fibers of $\Psi$ ).

Definition 5.1 Let $(P, Q, \rho) \in \Omega$ with $\operatorname{sh}(P)=\operatorname{sh}(Q)=\lambda$ and for $1 \leqslant i \leqslant \ell(\lambda)$ define $\mathbf{r}=\left(r_{i}\right)$ be the shift vector as in Section 4.2 Let $i_{1}$ be the smallest index for which $\lambda_{i_{1}+1}<\lambda_{1}$, $i_{2}$ be the smallest index for which $\lambda_{i_{1}+i_{2}+1}<\lambda_{i_{1}+1}$, etc. Reorder the first $i_{1}$ coordinates of $\rho-\mathbf{r}$ to be increasing, then reoder the next $i_{2}$ to be increasing, etc. Add $\mathbf{r}$ to the resulting vector to obtain a vector $\rho^{\prime}$ which we call the dominant representative of $\rho$.

Theorem 5.2 Let $(P, Q, \rho) \in \Omega$. Then $\Phi(\Psi(P, Q, \rho))=\left(P, Q, \rho^{\prime}\right)$.

As a corollary we can describe the fibers $\Psi^{-1}(w)$.

Corollary 5.3 Suppose $P$ and $Q$ are two tabloids with the same shape $\lambda$; let $r=\left(r_{1}, \ldots, r_{\ell(\lambda)}\right)$ be the vector of shifts described in Section 4.2. Let $S$ be the parabolic subgroup of the permutation group $S_{\ell(\lambda)}$ generated by transpositions $s_{i}$ such that $\lambda_{i}=\lambda_{i+1}$. Then $\Psi(P, Q, \rho)=\Psi\left(P, Q, \rho^{\prime}\right)$ if and only if $\rho-\mathbf{r}=s\left(\rho^{\prime}-\mathbf{r}\right)$ for some $s \in S$.

\section{Asymptotic realization via the usual Robinson-Schensted inser- tion}

In this section we describe how AMBC is related to the usual bumping algorithm. Any $w \in \widetilde{A}_{n-1}$ can be presented as an infinite semi-periodic sequence of integers:

$$
\ldots, w(-1), w(0), w(1), w(2), \ldots,
$$

where $w(i+n)=w(i)+n$. One can then apply the usual Robinson-Shensted algorithm to insert this sequence, choosing the initial place arbitrarily, for example at $w(1)$. 
Example 6.1 Let $n=6$ and let $w=[-4,5,-2,7,3,6]$. Then the sequence of $w(i), i \geq 1$ looks as follows: $-4,5,-2,7,3,6,2,11,4,13,9,12,8, \ldots$ Inserting this sequence, we obtain a sequence of tableaux

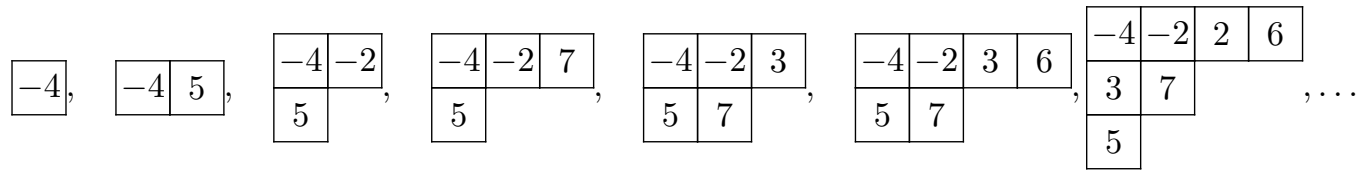

Denote $\bar{P}_{i}(w)$ the tableau obtained by inserting $w(1), \ldots, w(i)$. One can then create the associated tabloid $P_{i}(w)$ by passing from each number to its residue modulo $n$ and then forgetting the order of elements in each row.

Example 6.2 The above sequence of tableaux results in the following sequence of tabloids.

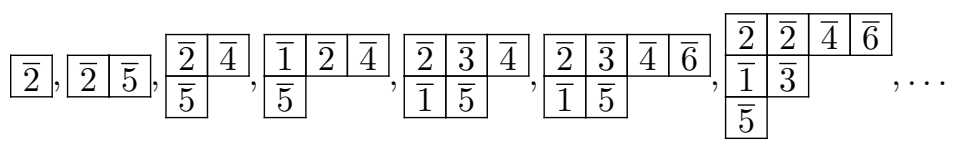

Theorem 6.3 For large enough $i$ we have

$$
P_{i+n}(w)=P_{i}(w)+P(w)
$$

where $P(w)$ is the tabloid obtained by Shi insertion of $w$, and content of tabloids is added as multisets: first row to first row, second row to second row, etc.

\section{References}

[Ari99] S. Ariki, Robinson-Schensted correspondence and left cells, arXiv:math/9910117, 1999.

[BV82] D. Barbasch and D. Vogan, Primitive ideals and orbital integrals in complex classical groups, Math. Ann. 259 (1982), 153-199.

[Chm15] M. Chmutov, Affine Matrix-Ball Construction Java program, https://sites.google. com/site/affiners/2015.

[CPY15] M. Chmutov, P. Pylyavskyy, and E. Yudovina, Matrix-Ball Construction of affine RobinsonSchensted correspondence, arXiv:1511.05861, 2015.

[Ful97] W. Fulton, Young tableaux: with applications to representation theory and geometry, London Mathematical Society Student Texts, vol. 35, Cambridge University Press, 1997.

[GM88] A. M. Garsia and T. J. McLarnan, Relations between Young's natural and the Kazhdan-Lusztig representations of $S_{n}$, Advances in Mathematics 69 (1988), no. 1, 32-92.

[Hon05] T. Honeywill, Combinatorics and Algorithms Associated with the Theory of Kazhdan-Lusztig Cells, Ph.D. thesis, University of Warwick, 2005. 
[KL89] D. Kazhdan and G. Lusztig, Representations of Coxeter groups and Hecke algebras, Invent. Math. 53 (1989), 165-184.

[Lus89] G. Lusztig, Cells in affine Weyl groups, IV, J. Fac. Sci. Univ. Tokyo Sect. IA Math. 36 (1989), no. 2, 297-328.

[Shi86] J. Y. Shi, Kazhdan-Lusztig cells of certain affine Weyl groups, Lecture Notes in Mathematics, vol. 1179, Springer-Verlag, 1986.

[Shi91] J.Y. Shi, The generalized Robinson-Schensted algorithm on the affine Weyl group of type $A_{n-1}$, Journal of Algebra 139 (1991), no. 2, 364-394.

[Vie77] G. Viennot, Une forme géométrique de la correspondance de Robinson-Schensted, Combinatoire et représentation du groupe symétrique (1977), 29-58.

[Xi02] N. Xi, The Based Ring of Two-Sided Cells of Affine Weyl Groups of Type $A_{n-1}$, Mem. Amer. Math. Soc., vol. 749, American Mathematical Soc., 2002. 
